\title{
ANDES

\section{Factores limitantes de la carga de entrenamiento físico en pandemia COVID-19}

\author{
Limiting factors of the physical training load in the COVID-19 pandemic
}

\author{
Héctor Fuentes-Barria $^{\oplus a}$, Raúl Aguilera-Eguía ${ }^{\circledR b}$, Catalina González-Wong ${ }^{\oplus}$
}

\begin{abstract}
aPrograma Magíster en Ciencias de la Actividad Física y Deportes Aplicadas al Entrenamiento, Rehabilitación y Reintegro Deportivo, Facultad de Salud Universidad Santo Tomas, Chile

'Departamento de Salud Pública, Facultad de Medicina, Carrera de Kinesiología. Universidad Católica de la Santísima Concepción. Concepción, Chile

cPrograma Magíster de Gestión en Salud, Facultad de Salud, Universidad del Desarrollo. Santiago, Chile
\end{abstract}

\section{Sr. Editor,}

Recientemente leímos con interés la carta editor titulada "Dosificación de la intensidad del ejercicio físico durante la cuarentena COVlD-19: ¿Funciona la autorregulación perceptiva en los niños?.” y su réplica denominada "COVID-19, cuarentena, intensidad del ejercicio y niños" ${ }^{1,2}$. Felicitamos a Rodríguez-Núñez quien aporta con la Escala EPInfant como una novedosa herramienta que permite controlar la intensidad de la carga gatillando beneficios del entrenamiento físico sobre la salud ${ }^{1}$. Además de Wiwanitkit quien comenta valiosos puntos relacionados con el tipo de actividad al momento de prescribir actividad física, ejercicio físico y deporte en contextos de cuarentena ${ }^{2}$. No obstante, con el objetivo de entregar mayor e importante información nos gustaría complementar en base algunas recomendaciones la información entregada en ambas cartas publicadas anteriormente.

Para realizar nuestros comentarios primero debemos recordar que la carga de trabajo físico está conformada por tres componentes básicos (intensidad, volumen y densidad) que independientemente del contexto definen y condicionan los beneficios del ejercicio sobre la salud, siendo la recomendación de la Organización Mundial de la Salud realizar un mínimo 60 min diarios de actividades de intensidad moderada o un mínimo de tres veces por semana de actividades a intensidad alta en niños y adolescentes de entre 5 y 17 años $^{3}$. Respecto al tipo de actividad la evidencia sugiere no limitar la realización de actividades grupales a espacios cerrados y abiertos sino más bien atenerse al concepto de distancia social considerando un mínimo $5 \mathrm{~m}$ para actividades de intensidad moderada y al menos $10 \mathrm{~m}$ para actividades intensas que involucren rápidos desplazamientos $(>14,4 \mathrm{~km} / \mathrm{h})$ en cualquier eje de movimiento ${ }^{4}$. En este sentido, la distancia social relacionada con el factor socioeconómico dependiendo del contexto no sería un limitante, puesto que algunos países como por ejemplo Chile permiten realizar actividades al aire libre a pesar de las cuarentenas ${ }^{5}$. Finalmente, esperamos estos comentarios contribuyan a complementar la información sobre la prescripción y practica de actividad física, ejercicio y deporte en población pediátrica en tiempos de cuarentena por COVID-19.

Correspondencia:

Héctor Fuentes-Barria

hectorfuentesbarria@gmail.com 


\section{Referencias}

1. Rodríguez-Núñez I. Dosificación de la intensidad del ejercicio físico durante la cuarentena COVID-19: ¿Funciona la autorregulación perceptiva en los niños? Rev Chil Pediatr. 2020; 91 (2): 304-5.

2. Wiwanitkit V. COVID-19, cuarentena, intensidad del ejercicio y niños. Rev Chil
Pediatr. 2020;91(6):1000.

3. Organización Mundial de la

Salud. Estrategia mundial sobre régimen alimentario, actividad física y salud. Actualización 2021. Disponible en: https://www. who.int/dietphysicalactivity/ publications/9789241599979/es/

4. Kalazich Rosales C, Valderrama Erazo P, Flández Valderrama J, et al. Orientaciones
Deporte y COVID-19: Recomendaciones sobre el retorno a la actividad física y deportes de niños niñas y adolescentes. Rev Chil Pediatr. 2020;91(7):75-90.

5. Biblioteca del Congreso Nacional de Chile (BCN). Resolución 43 Exenta. Capitulo II. Medidas plan "Paso a Paso". II Paso 1: Cuarentena. 2021. https://www.bcn.cl/ leychile/navegar?idNorma $=1154619 \&$ idPa rte $=10192645$. 